\title{
PREPARATION FOR NERVE MEMBRANE POTENTIAL READINGS \\ OF A LEECH, LABORATORY SETUP AND \\ DISSECTION PROCESS
}

\author{
A Thesis \\ Presented to \\ The Faculty of California Polytechnic State University, \\ San Luis Obispo
}

\begin{abstract}
In Partial Fulfillment
Of the Requirements for the Degrees

Master of Science in Engineering, with Specialization in Biomedical Engineering,

Bachelor's of Science in Electrical Engineering
\end{abstract}

by

Jason Patrick Caulfield

June 2009 
(C) 2009

Jason Patrick Caulfield

ALL RIGHTS RESERVED 


\section{Committee Membership}

TITLE:

PREPARATION FOR NERVE MEMBRANE

POTENTIAL READINGS OF A LEECH,

LABORATORY SETUP AND DISSECTION

PROCESS

AUTHOR: Jason Patrick Caulfield

DATE SUBMITTED:

June 2009

COMMITTEE CHAIR:

Robert Szlavik, Dr. and Professor

COMMITTEE MEMBER:

Lanny Griffin, Dr. and Professor

COMMITTEE MEMBER:

Robert Crockett, Dr. and Professor 


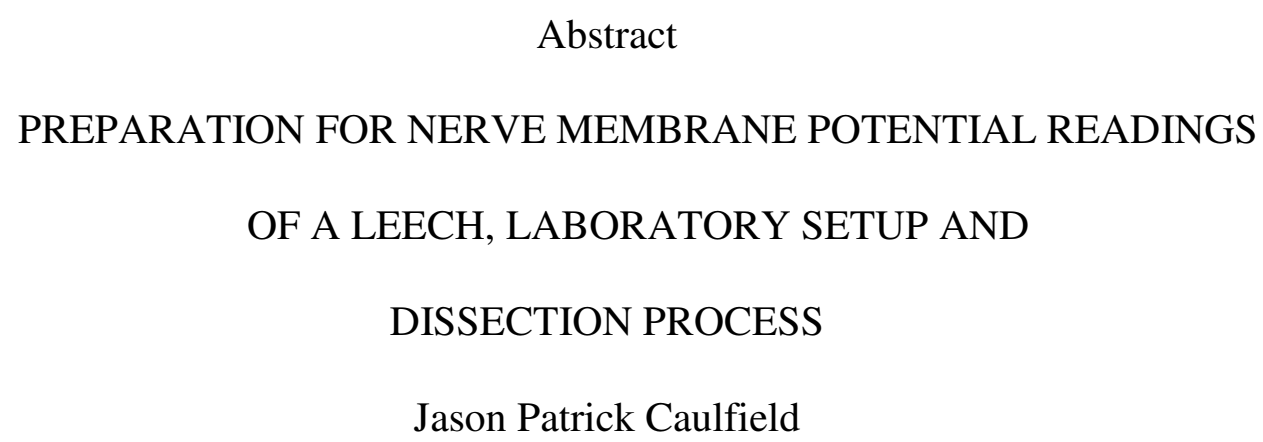

A well documented laboratory setup, leech preparation process, and bio-potential data recording process are needed. Repeatability and quality data recordings are essential and thus dictate the requirements of the laboratory setup and processes listed above. Advances in technology have both helped and hindered this development. While very precise equipment is required to record the low voltage bio-potentials, noisy electronic equipment and wires surrounding the work area provide high levels of interference. Proper laboratory setup and data recording processes, however, limit the unwanted interference. Quality data can only be recorded from a properly handled and prepared leech subject. Proper setup and procedures result in quality recordings which lend a clean signal for furthering the understanding of nerve functionality. The electrophysiology lab at California Polytechnic State University in San Luis Obispo is an example of a proven lab setup for high quality signal capture.

Keywords: leech, action-potential, laboratory, bio-potential, Hodgkin, Huxley, axon, nerve, ganglia, neuron, Calpoly, voltage-gated-ion-channels. 


\section{Acknowledgments}

\section{Dr. Szlavik}

I would like to thank Dr. Robert Szlavik for giving me the guidance and leadership that I needed in this new and exciting field of Biomedical Engineering. Without Dr. Robert Szlavik's help I would not have been able to complete this thesis project.

\section{CalPoly}

I would like to thank the CalPoly faculty and board for believing in the abilities of us, the student body. The lessons learned by the students through the opportunities provided last a lifetime and echo in our achievements.

\section{St. Jude Medical}

I would like to thank St. Jude Medical for providing the students of CalPoly with a great facility for housing the electrophysiology lab where my project was conducted. 
Table of Contents

Page

LIST OF TABLES $\ldots \ldots \ldots \ldots \ldots \ldots \ldots \ldots \ldots \ldots \ldots \ldots \ldots \ldots \ldots \ldots$ viii

LIST OF FIGURES $\ldots \ldots \ldots \ldots \ldots \ldots \ldots \ldots \ldots \ldots \ldots \ldots \ldots \ldots \ldots \ldots$ CHAPTER

I. $\quad$ INTRODUCTION $\ldots \ldots \ldots \ldots \ldots \ldots \ldots \ldots \ldots \ldots \ldots \ldots \ldots \ldots$

II. $\quad$ LEECH ANATOMY AND BACKGROUND $\ldots \ldots \ldots \ldots \ldots \ldots \ldots$

LEECH ANATOMY ................ 2

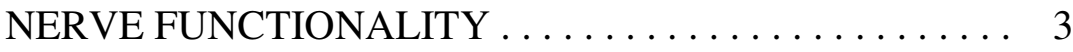

VOLTAGE CLAMPING ............. 5

III. $\quad$ LABORATORY SETUP $\ldots \ldots \ldots \ldots \ldots \ldots \ldots \ldots \ldots \ldots \ldots$

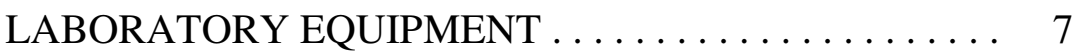

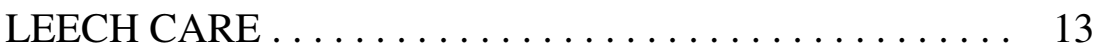

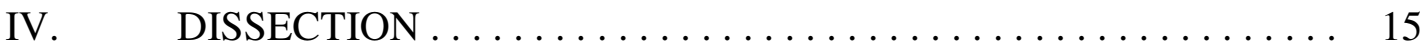

OBJECTIVE AND PREPARATION $\ldots \ldots \ldots \ldots \ldots \ldots$

STEP-BY-STEP DISSECTION PROCESS . . . . . . . 17

V. MEMBRANE POTENTIAL RECORDING PROCEDURES . . . . . 19

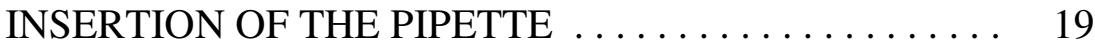

EQUIPMENT READINGS . . . . . . . . . . . 19

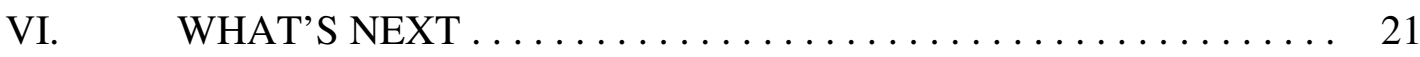

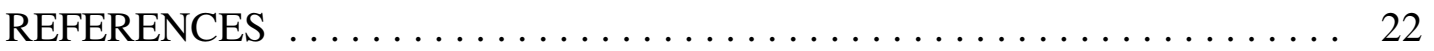


Table of Contents Continued

Page

APPENDICES

A. LEECH CLASSIFICATION AND FEEDING BEHAVIORAL ................ 23

B. LEECH ANATOMY DIAGRAMS ........... 24

C. GANGLION MAP AND INDEX ........... 25

D. ADDITIONAL GANGLION NOTES .......... 28

E. SIMPLIFIED NERVE DIAGRAM . . . . . . . . . 29

F. LABORATORY LIST OF MATERIALS AND 30

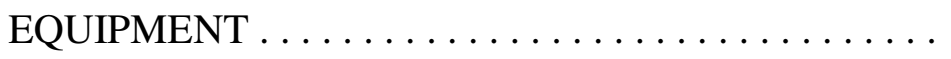

G. LABORATORY EQUIPMENT CONNECTION 31

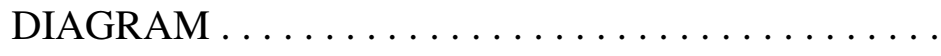

H. PIPETTE PULLING SETTINGS . . . . . . . . . . 32

I. STEP-BY-STEP PROCURES FOR DISSECTION OF A 33 GANGLIA $\ldots \ldots \ldots \ldots \ldots \ldots \ldots \ldots \ldots \ldots \ldots$

J. TEST CELL SCHEMATIC . ............ 34

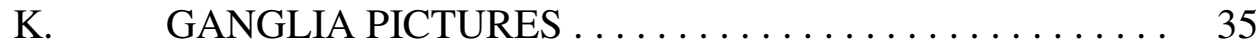

L. DARK FIELD MICROSCOPY OF SINGLE GANGLION. . 36 


\section{List of Tables}

Table

1. Characterization of three leech orders $\ldots \ldots \ldots \ldots \ldots \ldots \ldots \ldots \ldots$

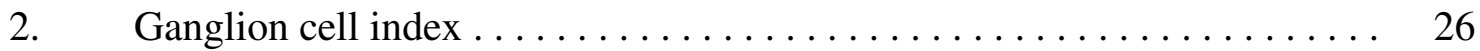

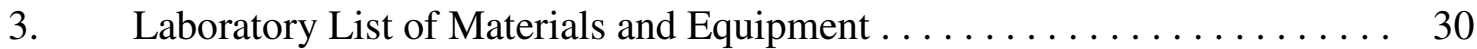

4. Settings for pulling pipettes with the Flaming/Brown Micropipette Puller. . 32 


\section{List of Figures}

Figure Page

1. Diagram of a leech cross-section $\ldots \ldots \ldots \ldots \ldots \ldots \ldots \ldots \ldots \ldots \ldots$

2. Depiction of an action potential $\ldots \ldots \ldots \ldots \ldots \ldots \ldots \ldots \ldots \ldots$

3. Simple diagram of a voltage clamping apparatus $\ldots \ldots \ldots \ldots \ldots$

4. Graphical representation of the $\mathrm{Ag}-\mathrm{AgCl}$ and $\mathrm{KCl}$ reaction. . . . . . . . 9

5. Diagram of leech pinning process $\ldots \ldots \ldots \ldots \ldots \ldots \ldots \ldots \ldots$

6. Full length leech anatomy diagram ................ 24

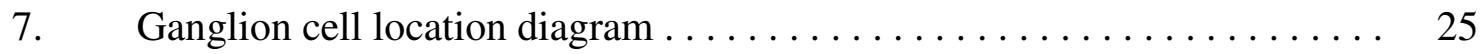

8. Simplified nerve diagram labeling key parts . . . . . . . . . . . . . 29

9. Wiring diagram of data acquisition hardware $\ldots \ldots \ldots \ldots \ldots \ldots \ldots$

10. Axon instruments test cell schematic. ............... 34

11. Group of ganglia, single ganglia, and single ganglia with sheath removed. . 35

12. Dark field microscopy of single ganglion. . . . . . . . . 36 


\section{CHAPTER I}

\section{Introduction}

Day to day "modern man" experiences a simple partnership with electrical equipment mainly providing comfort, entertainment, and communication. However, a trip to the hospital requires a deeper relationship. The fundamental marriage of electronics and human physiology starts with the action potential of a nerve. Knowledge of the dynamics of nerves is the foundation that scientists and engineers need to design equipment destined to better lives. The experimental setup below is designed to aid in building this knowledge.

Like all experiments it is important for a setup that allows for repeated testing under controlled conditions. For this reason alone this paper focuses on the testing of nerves within leeches which can be easily duplicated in a small laboratory and with little equipment. Below you will find the background knowledge needed to care for leeches as well as the anatomical understanding required to perform and analyze experiments on leech nerves. This paper provides a detailed explanation of the electrophysiology lab at California Polytechnic State University in San Luis Obispo. The step by step procedures listed here are for the dissection of a leech and the insertion of an electrode into a leech nerve.

It is the knowledge communicated in this paper that allows engineers and scientists to take steps leading to tomorrow's technology. However, before any step is taken the basics must be understood and for this report the basics start with an understanding of leech neurophysiology. 


\section{CHAPTER II}

\section{Leech Anatomy and Background}

\section{Leech Anatomy}

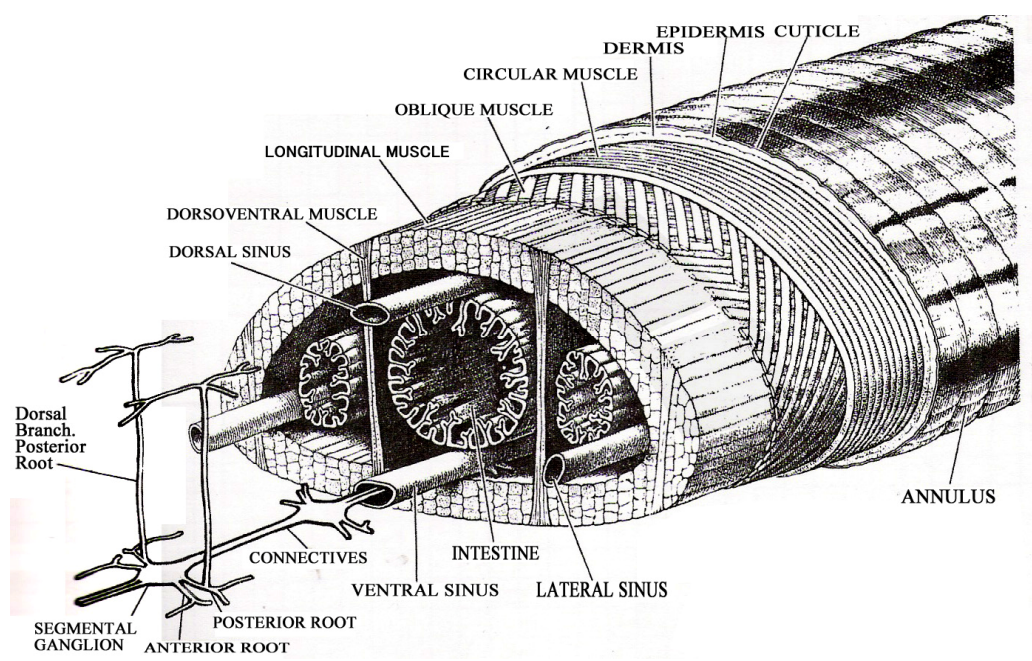

Figure 1. Diagram of a leech cross-section [1].

Leeches, Hirudinea class, are related to earthworms under the super class

Clitellata. There are several orders and families of distinct leeches. Leeches are distinct mainly by their habitat, size, behavior, and feeding habits [1]. Some classification distinctions and general notes can be found in appendix A.

Leeches have several anatomical layers which are depicted in Figure 1. The tough outer layer called the cuticle has the main purpose of protection. Directly under the cuticle are the epidermis and dermis. Below these layers, four layers of muscle provide the leech with great maneuverability and elasticity. Within the circular housing of the muscles and dermis layers, four sinuses and the intestine run the length of the leech. Two lateral sinuses provide blood circulation and exhibit a rhythmic pumping fulfilling the same function as the human heart. The ventral sinuses house the nerves and the ganglion nerve junctions [1]. A graphical representation of the cross-section of a leech is found in appendix B. 
Nerves are made of many neurons. Nerves branch from within the ganglia and terminate locally. This arrangement gives each ganglion control over a small part of the leech body. Twenty-nine ganglia are dispersed throughout the leech body and work in collaboration. One ganglion is located in the head, seven are in the tail, and 21 are evenly dispersed down the center of the leech [1].

Inside each ganglion, neurons intersect and interact. Inside the clear, jelly-like ganglion, the neuron junctions form an organized pattern. The consistency of location allows for the mapping of the neurons in leech ganglia [1]. Such a map is found in appendix $\mathrm{C}$ and additional notes on ganglia are found in appendix D.

Neurons are made of three main parts. The Soma, which can be seen under a microscope, is the cell body and houses the nucleus. Dendrites branch out from the Soma and receive signals from other neurons. The axon is thin and stretches out over relatively long distances. The end of the Axon not connected to the Soma branches out stimulating other neurons or muscle fibers [2]. Appendix E is a graphical diagram of a nerve.

\section{Nerve Functionality}

Ion concentrations inside and outside neurons are different and thus produce an electrical potential across the membrane. Prior to a stimulus the membrane voltage or potential is a DC value and can be calculated with the Goldman Equation. This equation considers the charge and consentration of ions permiable to the cellular membrane. The equation results in a resting membrane potential. For most neurons this potential is approximately $-50 \mathrm{mV}$. A change in ion concentrations changes the membrane voltage [3]. 
An influx of positively charged ions to the cell results in an increase in the membrane voltage. Conversely, an efflux of positively charged ions to the cell results in a decrease of the membrane voltage. The influx and efflux of negatively charged ions result in converse membrane voltage changes to that of positively charged ions. Under resting circumstances, all of the actions above are constantly occurring negating one another. However, a net movement of ions is the result of a stimulus.

Once stimulated, neurons change the voltage across their membrane. This voltage change is in response to an excitation of one or more Dendrites. When a Dendrite is excited, it changes the concentration of ions inside the neuron local to the excited Dendrite. Consequently this phenomenon changes the membrane potential local to the excited Dendrite. Once a threshold membrane voltage is reached, voltage-gated ion channels are activated. Voltage-gated ion channels, as the name suggests, trigger at preset membrane voltage levels. When triggered, these gates open and transfer ions across the membrane.

When enough Dendrites are excited, the membrane voltage level in the Soma and Axon trigger a cascading effect of opening and closing gates causing the signal to propagate down the axon. As the result of various voltage-gated ion channels working together, the propagated signal down an Axon of a neuron is a highly distinguishable wave pattern [3]. 


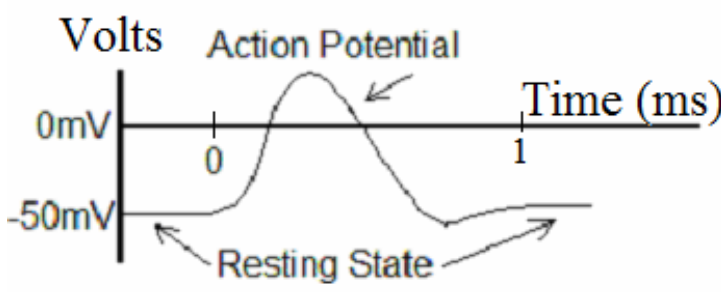

Figure 2. Depiction of an action potential.

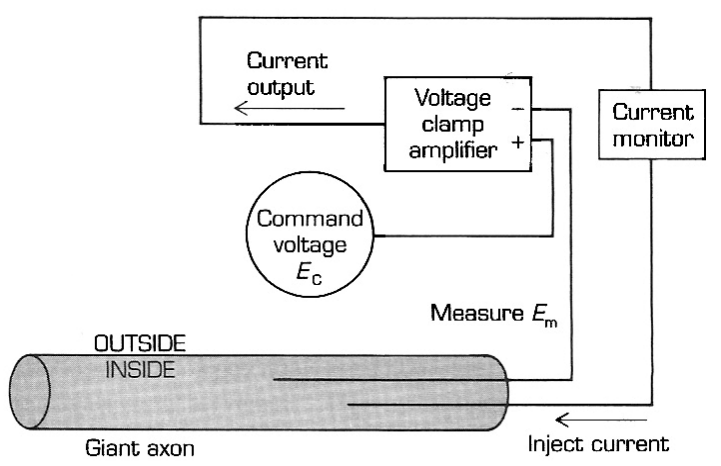

The distinguishable membrane potential pattern or signal resulting from a stimulus is shown in Figure 2. The frequency of the signal is dependant on the intensity of the stimulus; However, the signal shape and amplitude are independent of the stimulus. The signal propagates the length of the Axon resulting in the stimulation of other neurons or muscular tissue [3]. To record the membrane potential, an electrode must be inserted into the Soma or Axon. This proves difficult due to the tiny diameters of most axons. Alan L. Hodgkin and Andrew F. Huxley in the 40's found that the giant Axon of a squid made testing much easier.

\section{Voltage Clamping}

Hodgkin and Huxley, through their experiments, developed a process, called voltage clamping, to measure the flow of ions across the membrane of a neuron. This process allows for the characterization of the voltage-gated ion channels. As a function of the present membrane voltage, the voltage-gated channels transport ions across the membrane thus, under normal conditions, changing the membrane voltage. However, by injecting or removing charge from the intercellular fluid, with an electrode to negate the effects of the voltage-gated ion channels, the membrane voltage is clamped at a constant 
value. Monitoring the required influx or efflux of current, at various voltage levels, allows for the characterization of the electrical properties of the cell [2],[3]. Figure 3 shows a diagram of this process. The quality of the data is determined by the quality of the laboratory and specimen setup prior to collecting data. 


\section{CHAPTER III}

\section{Laboratory Setup}

\section{$\underline{\text { Laboratory Equipment }}$}

Selecting the appropriate equipment is highly important and should be customized to the type of research being conducted. A complete list of lab equipment and materials is found in appendix F. The electrophysiology lab at California Polytechnic State University in San Luis Obispo (CalPoly) utilizes three data acquisition instruments which are all connected to a single desktop computer. A complete wiring diagram is found in appendix G. Each data acquisition instrument has a unique purpose. The National Instruments (NI) PCI6259 data acquisition board can be controlled with Lab View software which allows for signal generation, real-time recording, and real-time processing of bio-potentials including the use of various types and degrees of low-pass, high-pass band-pass, and notch filters. The NI data acquisition board has 32 analog inputs and four analog outputs. The 32 inputs can be configured as differential inputs dividing the number of inputs by two. This laboratory does not use the differential setup because the bio-potentials are not read directly by the NI hardware and therefore differential readings are not needed. The NI data acquisition I/O's are connected to the Axon Instruments Digidata 1440A. The Digidata has a custom graphical user interface (GUI) software package allowing for similar functions as Lab View, however, it does not allow for complex real-time digital signal processing (i.e. it only performs simple highpass and low-pass filtering). The Digidata 1440A can acquire 16 analog signals, output eight analog signals, and output four digital signals.

Designed to interface directly with the Digidata 1440A, the Axon Instruments MultiClamp 700B directly acquires bio-potentials through two separate headstages. 
Controlled through a limited computer GUI command center, the MultiClamp 700B has two inputs from two calibrated headstages and two digital inputs (labeled "Command") which control each headstages' functions. The headstages can be switched between current-clamp and voltage-clamp modes via the one bit digital inputs or via the MultiClamp 700B computer interface. The MultiClamp 700B has three outputs for each of the 2 acquired signals. These outputs are labeled: "Primary," "Secondary," and "Scope." Simple filtering can be applied to each output channel of the MultiClamp 700B (e.g. low-pass filtering and highpass filtering.) Compensation filtering can be applied to each headstage to account for offset voltages and stray capacitances of the electrodes.

Each headstage has a silver/silver-chloride $(\mathrm{Ag}-\mathrm{AgCl})$ wire electrode created by bathing a $0.999 \%$ silver wire for 30 minutes in a high concentration chlorine bath (e.g. bleach). This process increases the conductivity between the electrode and the surrounding fluid. After bathing, the electrode is inserted into a glass pipette filled with a $0.3 \mathrm{M} \mathrm{KCl}$ solution and connected to the headstage. A positive electrical gradient between the $\mathrm{KCl}$ solution and the $\mathrm{Ag}-\mathrm{AgCl}$ wire causes $\mathrm{AgCl}$ to convert back to $\mathrm{Ag}$ releasing a $\mathrm{Cl}^{-}$ion thus creating current flow into the wire electrode from the solution. A negative electrical gradient between the $\mathrm{KCl}$ solution and the $\mathrm{Ag}-\mathrm{AgCl}$ wire causes free $\mathrm{Cl}^{-}$ions from the $\mathrm{KCl}$ solution to bond to exposed $\mathrm{Ag}$ wire producing a free electron inside the Ag wire thus creating current flow from the electrode to the solution. Figure 4 is a graphical representation of this reversible processes. 


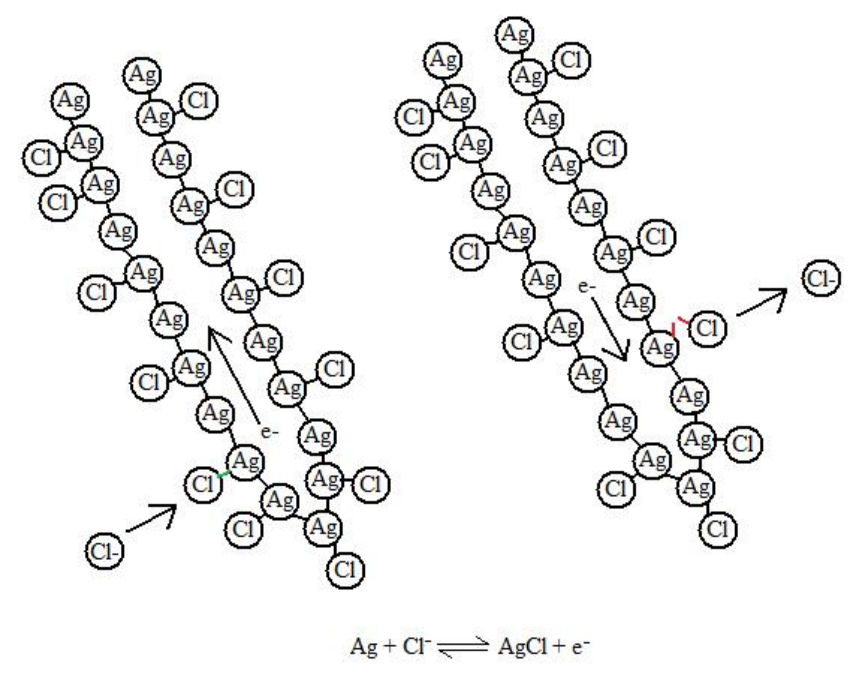

Figure 4. Graphical representation of electrode reaction.

Each electrode pipette, which house the $\mathrm{Ag}-\mathrm{AgCl}$ wire and $\mathrm{KCl}$ solution, is created in the CalPoly electrophysiology lab. The CalPoly electrophysiology lab utilizes the Flaming/Brown Micropipette Puller for pipette pulling. The pipettes are pulled prior to each experiment to limit the chance of the tip being broken while in storage. A broken tip will greatly increase the difficulty of piercing the cell membrane. The pipettes are pulled as sharp electrodes using a trough heating coil. The specific program settings for pulling $1 \mathrm{~mm}$ and $1.5 \mathrm{~mm}$ O.D. pipettes can be found in appendix H. With a MicroFil fine needle syringe purchased through World Precision Instruments Inc, each pipette is filled with a $\mathrm{KCl}$ solution. The pipettes are mounted to the headstages which are mounted on micro-positioning devices next to a compound Nikon FN1 microscope designed for electro-physiology applications.

The dissection of the specimen is best accomplished with a low power microscope, while utilizing a microscope with higher magnification for inserting the pipette into the cell. The dissection of the leech is performed using a dissection microscope with a magnification of 10x - 50x. To obtain a detailed view of a ganglion and the nerve cells 
inside, a high power compound Nikon FN1 microscope setup for dark field imaging is used with a magnification of 250x to 500x. The Nikon microscope is mounted on a Gibraltar table atop a vibration dampening table.

The vibration dampening table utilizes air cushioning to limit the vibrations of the specimen, the microscope, and the pipette electrodes. The Nikon microscope mounted to the Gibraltar Table allows for the microscope to move while the specimen remains stationary, this further prevents unnecessary vibrations of the specimen as the microscope position and magnification are adjusted. The Nikon microscope, Gibraltar Table, and vibration dampening table are located inside a Faraday Cage.

Low noise readings can only be performed in a Faraday Cage. A Faraday Cage is a large grounded metal box or cage designed to block out Electromagnetic interference. Electromagnetic signals (e.g. outside electrical noise) that come in contact with an average wall are influenced by the material characteristics of the wall. In almost all cases, the signal suffers attenuation but is able to propagate through the wall. However, if that same electromagnetic signal comes in contact with a grounded metal wall (e.g. any side of the Faraday Cage,) the wall maintains a constant electrically-grounded state creating a ground plane boundary condition effectively "shorting" the signal to ground preventing it from propagating through the wall. This limits outside electrical noise from interfering with precise, low voltage readings captured within the cage. All experimental data is collected from within the Faraday Cage. All electrical equipment inside the Faraday Cage are shielded and grounded to prevent interference with nerve signals. The CalPoly electrophysiology lab Faraday Cage is a $12 \times 8 \times 8$ foot box with a large steel door. The cage houses all of the equipment except the computer and equipment used for dissection. All noise sensitive measurements of bio-potentials are read with the door closed and 
sealed. A single shielded fan and filter refreshes the air inside the cage for operator safety. The purpose of the Faraday Cage is to shield the inside environment from external electrical noise.

Electrical wires and devices inside the Faraday cage are sources of noise. The large amplitude noise in the cage may over power the desired signal from the nerves. The amplitude of the signal from the nerves is approximately $100 \mathrm{mV}$ as compared to possibly 200 - $300 \mathrm{mV}$ amplitude of the noise when unshielded and unfiltered, as received through a headstage and read from an oscilloscope. The largest amplitude noise is narrow-band centered at $60 \mathrm{~Hz}$. Noise content is either broadband or narrow-brand. Broadband noise spans a large frequency range. Narrow-band noise exists over a small number of frequencies. Broadband noise is limited by proper lab setup such as limiting the amount and types of electrical equipment in the faraday cage, completely shielding noise sources, and proper setup of the pipette electrodes. Narrow-band noise is limited through the same processes as the broadband noise as well as with software notch filters and electronic notch filters built into the data acquisition boards.

Cell phones and other hand-held devices emit broadband noise. These devices are mainly housed in plastic and therefore have no shielding to block their broadband noise interference; the noise from these devices are eliminated by keeping them outside of the Faraday cage. Most other equipment such as the Axon Instrument's MultiClamp 700B and microscope lamp houses are shielded because of their metal cases. These metal cases are grounded by connecting a wire from the case to the metal wall of the Faraday cage. This keeps the noise inside of the metal case and away from the electrodes. Electrical wires installed by licensed electricians are shielded by the metal conduit that houses the wires. However, cables used to power equipment are not shielded. These cables emit 
narrow-band noise with all content centered around $60 \mathrm{~Hz}$. Filters eliminate the noise from the cables.

Both broadband and narrow-band noise is further limited by setting up the pipette electrode properly. A proper electrode wire, within the pipette, has a length of which the tip is located as closely as possible to the tip of the pipette without clogging the pipette. Increasing the distance from the electrical source (i.e. the cell under test) and the electrode wire increases the series resistance in the path of the signal. Pushing the wire too far into the pipette does not allow for adequate conductivity between the electrode and the solution at the tip of the pipette. Conductivity is proportional to the surface area of the wire that receives the biological signal from the surrounding solution. Clogging the tip of the pipette does not allow the signal to propagate through the solution in the pipette, thus limiting the conductive surface area to only the tip of the wire, effectively producing a large series resistance. As either case listed above result in an increased series resistance and antennas, in which the series resistance is close to infinite, are highly conducive to capturing noise; it stands that minimizing the series resistance minimizes noise. Therefore, proper electrode length minimizes the influence of noise.

Test cells, provided by Axon Instrument, are used for training, calibration, and noise detection. Modeling three different methods of measuring bio-potentials, the test cell has four ports. One port acts as a ground or solution contact. The other three ports are "bath", "cell", and "patch". The "bath" simulates an electrode inserted into the liquid bath around the specimen. The "cell" port models the insertion of an electrode through the cell membrane. The "patch" port models an electrode on the surface of a cell which requires a Giga-seal to obtain readings of the membrane potential [8]. This lab setup is modeled using the "cell" port of the test cell. The test cell circuit diagram is appendix J. 


\section{$\underline{\text { Leech Care }}$}

Leech USA, LTD provides healthy and sterile leeches. Using sterile leeches, which prevents them from having diseases, is important in the event of a bite on a human. Along with the leeches, Leech USA, LTD provides a salt mixture used to create Ringer's solution which is an osmotic balanced solution for the leeches to live in. Ringer's solution is created by diluting $2 \mathrm{~g}$ of the salt mixture with $1 \mathrm{gal}$ of water creating an environment that is healthy for leeches. When the leeches are kept in a refrigerator, the water is changed weekly; otherwise, the water is changed more frequently. Lowering the temperature of the leeches decreases the leeches' metabolism which decreases their activity, decreasing their need to feed. Storing the leeches in a regular refrigerator allows the leeches to survive for $6-8$ months without a feeding. As leeches are very susceptible to temperature shock, the leeches are transferred to water that is already at the same temperature as the water they have been living in. This lab utilizes a Leech Mobile Home purchased through Leech USA, LTD which provides for easy water changing. A cheaper alternative is glass jars covered with cheese paper. Cheese paper allows for air to circulate through the jar, but does not allow leeches to escape.

To prepare leeches for surgery, they are anesthetized in a small uncovered beaker. First, the beaker is filled halfway with water at the same temperature as that which the leech has been living in, thus preventing temperature shock. The leeches are then inserted into the beaker. Two teaspoons of ethyl alcohol are then added to the solution. The beaker is allowed to sit at room temperature. This process causes the leech to become more animated moving violently in the confined space and often cause the leech to throw up blood. It is desired to have the leech expel the contents of its stomach as it decreases the amount of blood that must be cleaned up during surgery. After 30 minutes the leech 
is completely anesthetized and ready for surgery. This state is signified by the leeches' completely limp and non-active body. Completely anesthetized, the leeches are nonresponsive to the pinning process. If the leech does not exhibit these behaviors, the leech is given more time in the alcohol solution. 


\section{CHAPTER IV}

\section{Dissection}

\section{$\underline{\text { Objective and Preparation }}$}

The objectives of the leech dissection, for the protocol that was investigated, are to expose and prepare one or more ganglion for the insertion of a pipette electrode into one of the neurons located inside the ganglion. Inserting an electrode into a ganglion and recording data takes time; therefore, the leech must be prepared in such a way that the ganglion will continue to be a viable test subject for the entirety of the tests. Appendix K is pictures of exposed ganglia.

The instruments for the dissection include a pair of scissors, two forceps, a microscope, six pins, and a pinning tray. In the CalPoly laboratory, this process is completed under a dissection microscope with magnification between $10 \mathrm{X}$ and 50X. The leech is first prepared on a Petri dish.

The specimen is mounted on a Petri dish filled with Sylgard. Sylgard's elastic properties cause holes, created while pinning specimens, to automatically close. White wax is a good alternative. The wax is first melted and then pored into the dish creating an even surface. A simple lighter is used to re-melt the wax filling in holes created while pinning down the specimen. Small pins are used to pin down the leech. Micro-pins are used to pin the ganglion once removed from the leech. Micro-pins are purchased from Fine Science Tools and have a thickness similar to human hair. 


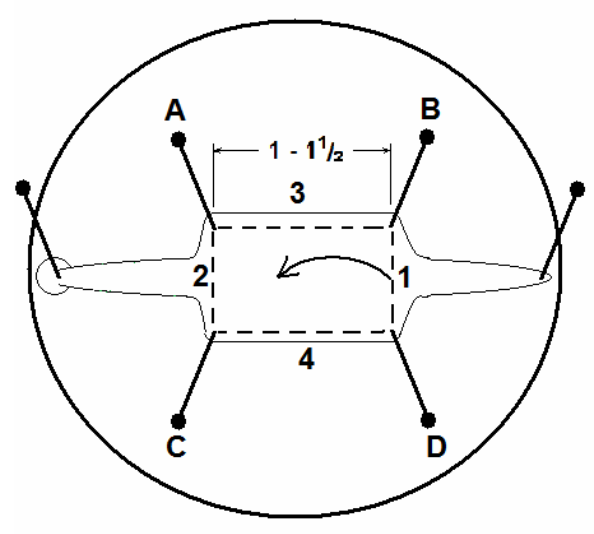

Figure 5. Diagram of leech pinning process.

The leech is pinned with the dorsal side facing up; this is the same as the dark green side up, the mouth facing downward, or the light green side facing downward. The leech is pinned to the tray using six pins as depicted in Figure 5. Two pins are used to pin down the head and tail while the remaining four are used to create a box in the center of the leech body. The leech is first pinned in the tail close to the edge of the tray and then the head is pinned at the opposite side of the tray stretching the leech. The leech body is usually taut. If the leech is longer than the tray, another pin close to one of the ends is added in which an "L" shape is made with the leech, making the dissection area taut.

The remaining four pins form a box shape in the middle of the leech. This box outlines the area of the dissection and is an appropriate length to include the number of ganglia to be exposed. The area inside the box is flat and taut making it easy to create incisions in the leech. More details are found in appendix L. 


\section{$\underline{\text { Step-by-Step Dissection Process }}$}

Once the leech is pinned to a tray with the dorsal side up, the leech is ready for dissection. The incisions in the specimen are made using stainless-steel scissors and forceps. A step-by-step process is found in appendix I. The dissection is performed under the dissection microscope using 10X to 50X magnification. The incisions are made in one of two ways. The first entirely removes the outer layer enclosed in the box. The second creates flaps that are re-pinned allowing for more muscle area within the dissection region. Both methods include a pair of incisions lateral or perpendicular to the length of the leech. These incisions start at one pin and move laterally across the leech terminating at the opposite pin. The two methods differ with the next incision(s). The first method continues with two incisions parallel to the dorsoventral axis or the length of the leech starting at one pin and ending at another. This completes incisions around the entire perimeter of the dissection area created by the pins. The other method includes only one incision along the dorsoventral axis making an 'I' shape. The two sections are folded back and repined making a larger box around the dissection area.

The above incisions cut through the dermis layers, the muscle layers, and the upper layer of the intestine. At this point, the ventral sinus housing the nerves is seen as a black line along the dorsovental axis. Using forceps, the clear, lower layer of the intestine is stretched until it tears. Using two forceps, the ventral sinus, or black sheath, is removed from around the clear ganglia. The ganglia are found by locating black lateral nerves that extend from the ganglion to muscle fibers. To prevent breaking the fragile nerves, the stress on the ganglia is minimized while peeling off the sinus sheath. Using two forceps allows for limiting the stress on the nerves. One forceps tip is pierced through the sheath. The other forceps grip the sheath and pull away from the tip of the 
other forceps. This action causes the sheath to tear and minimizes the stress on the nerves. Once the ganglion is fully exposed, the ganglion is extracted and pinned to another petri dish. A small amount of Ringer's solution is added around the ganglion and the leech to prevent them from drying out. The extracted leech ganglion is taken to a microscope with higher magnification for the insertion of pipette electrodes into the nerves of a ganglion. 


\section{CHAPTER V}

\section{Membrane Potential Recording Procedures \\ Insertion of the Pipette}

With a ganglion map, the desired nerve is located based on its general location and size with respect to the other nerves. A ganglion map is found in appendix C. Dark field microscopy makes the individual nerves more distinguishable.

A sharp pipette tip is imperative to the piercing of the ganglia sack and nerve itself. The quality of the pipette tip can be gauged by the impedance between the electrode and the bath solution. A sharp pipette tip is approximately $0.7 \mu \mathrm{m}$ in diameter with an impedance between 20M Ohms and 80M Ohms. The pipette is slowly moved toward the ganglion sack. A slight depression on the sack surface indicates contact with the ganglion. The sub-micron sized tip of the pipette is too small to be seen, thus it appears that the pipette tip has not yet touched the ganglion sack. Slowly and slightly moving the pipette toward and away from the ganglion sack punctures the sack, noticeable by the rapid change in the shape of the depression. In the case of the pipettes tip braking, the pipette is replaced immediately. On the surface of the sack, the electrical signal is large in amplitude, consisting only of noise, as the sack acts like an antenna. However, puncturing the sack makes the noise signal amplitude decrease as desired.

Successful insertion of the pipette into the nerve is indicated on the oscilloscope. The indicating signal on the oscilloscope is a negative DC offset of $50-70 \mathrm{mV}$. Noise amplitudes of $1-2 \mathrm{mV}$ are normal. Additional post recording digital filtering further decreases the strength of the noise. 


\section{Equipment Readings}

While working to place the pipette tip inside the nerve as well as during data gathering, it is important to have all of the equipment setup appropriately. The following section discusses the equipment found in the CalPoly electrophysiology lab as examples. During the experiment it is import to see all DC offsets. During an action potential from a P cell the signal amplitude is approximately $100 \mathrm{mV}$ with a frequency range of DC - 10 KHz. Noise amplitudes exceeding that of the action potential signal indicates that the pipette tip is in the wrong location, a need for additional filtering, or inadequate shielding of the test equipment from outside noise (e.g. cell phones, TV's, etc.).

The oscilloscope is used when trouble-shooting noise and inserting the pipette tip into the nerve. Minimizing the amplitude of the noise is done by monitoring the amplitude on the oscilloscope. While inserting the pipette tip, the oscilloscope provides fast and real-time measurements making it easy to see the rapid change in signal as the pipette punctures the cell.

Compensation for pipette internal resistances and capacitances are controlled via the Axon Instruments Digidata and MultiClamp software. After the pipette tip is inserted into the nerve cell, this software also measures the pipette tip impedance. With this software's simple band-pass filtering, noise outside of the desired frequency range is minimized. With the ability to voltage clamp the cell, this software is used to collect data points.

Clampex generates a current to voltage (I-V) characteristic curve by measuring the cells current draw when clamped under various voltages. Notch-filtering can be used in Clampex to further limit noise. 


\section{CHAPTER VI}

\section{What's Next?}

Tomorrow's life saving technology is the research of today. With the techniques described in this paper, today's research can expand the knowledge of leech neurophysiology. By drawing connections between human and leech nerves, this research expands the knowledge of human neurophysiology.

Replacing neural conductive pathways with discrete electrical components may produce a substitute for damaged nerves. The speeds of today's microcomputer processors far surpass the low frequency of action potentials. This feature makes it possible for one microcomputer to function in real-time in the place of many nerves. Much like silicon based components, nerves only relay a simple message from one point in the body to another. This paper is one building block of the bridge between cellular based nerves and silicon based electrical components.

The procedures built upon the techniques described in this paper may be used to verify the outcome of research in other disciplines. Growing nerves in a laboratory is cutting edge, but without tests to confirm their functionality they will never be practical for human use. Before tested on humans, the techniques in this paper give the ability to test and confirm that the experimental nerves function properly. It is only with this type of verification that multidisciplinary research can move forward into tomorrow's technology.

Whether these techniques are used to forge new paths or further support current engineering research, this paper ties the imaginations of engineers to the reality of tomorrow's life saving technology. Tomorrow's technology will make lives better; so in turn, today's engineering of that technology make lives better. 


\section{REFERENCES}

[1] Muller, Kenneth J, et al. Neurobiology of the Leech. Cold Springs Harbor, NY: Cold Spring Harbor laboratory, 1981.

[2] Guyton, Arthur and Hall, John. Textbook of Medical Physiology $5^{\text {th }}$ Ed, Philadelphia, PA: Elsevier Inc, 2006.

[3] Matthews, Gary G. Cellular Physiology of Nerve and Muscle $4^{\text {th }}$ Ed, New York: Blackwell Publishing, 2003.

[4] Axon Istruments. Axon CNS Guide to Electrophysiology \& Biophysics Laboratory Techniques $2^{\text {nd }}$ Edition. Computer software. CD-Rom. Union City, CA, 2006

[5] Axon Istruments. User Guide for Digidata 1440A. Computer software. CD-Rom. Union City, CA, 2006

[6] Fatt, P. and B. Katz. "An Analysis of the End-Plate Potential Recorded with an IntraCellular Electrode.” London: University College London, 1951.

[7] Kuffler, Damien P. "Neuromuscular Transmission in Longitudinal Muscle of the Leech.” Los Angeles, CA: University of California at Los Angeles, 1977.

[8] Molecular Devices Corporation. MultiClamp 700B Computer-Controlled Microelectrode Amplifier Theory and Operation. Computer software. CD-Rom. Union City, CA, 2005.

[9] National Instruments. DAQ M Series Manual. Computer software. CD-Rom. Austin, Tx, 2007.

[10] Slade, Carole. Form \& Style $12^{\text {th }}$ Ed. Boston, MA: Houghton Mifflin Company, 2003. 


\section{APPENDIX}

Appendix A: Leech Classification and Feeding Behavioral Notes [1]

\section{$\underline{\text { Classification }}$}

Clitellata Superclass

Oligochaeta Class (Earth Worm)

Hirudinea Class (Leech)

Rhynchobdellida Order

Piscicolidea Family (found in salt water)

Glossiphoniidae Family (found in fresh water)

Pharyngobdellida Order

Erpodbdellidae Family

Gnathobdellida Order

Hirudinidae Family (aquatic)

Haemadipsidae Family (terrestrial)

\begin{tabular}{l|l|l}
\multicolumn{1}{c|}{ Gnathobdellida } & \multicolumn{1}{|c}{ Phynchobdellida } & \multicolumn{1}{|c}{ Pharyngobdellida } \\
\hline Bloodsucking & Bloodsucking & Feed on small invertebrates \\
Jaws of teeth & Jaws of teeth & Swallow prey whole \\
Bite host & Bite host & Lack jaw \& teeth \\
$\begin{array}{c}\text { Block clotting in own gut } \\
\text { Use: Polypeptide } \\
\begin{array}{c}\text { Hirudin to inhibit } \\
\text { thrombin }\end{array}\end{array}$ & $\begin{array}{l}\text { Block clotting in host } \\
\text { Usementin to inhibit }\end{array}$ & \\
& thrombin &
\end{tabular}

Table 1. Characterization of three leech orders.

\section{$\underline{\text { Feeding Behavioral Notes }}$}

- Size increases with each feeding

- An inactive period follows each feeding

- lack responsiveness \& movement or activity

- rest in dark areas

- When hungry:

- stand up straight while swaying using mouth as anchor

- highly responsive to light \& mechanical stimuli

- frequently change location (moving toward water) 


\section{Appendix B: Leech Anatomy Diagrams}

Figure - Cross sectional of a leech

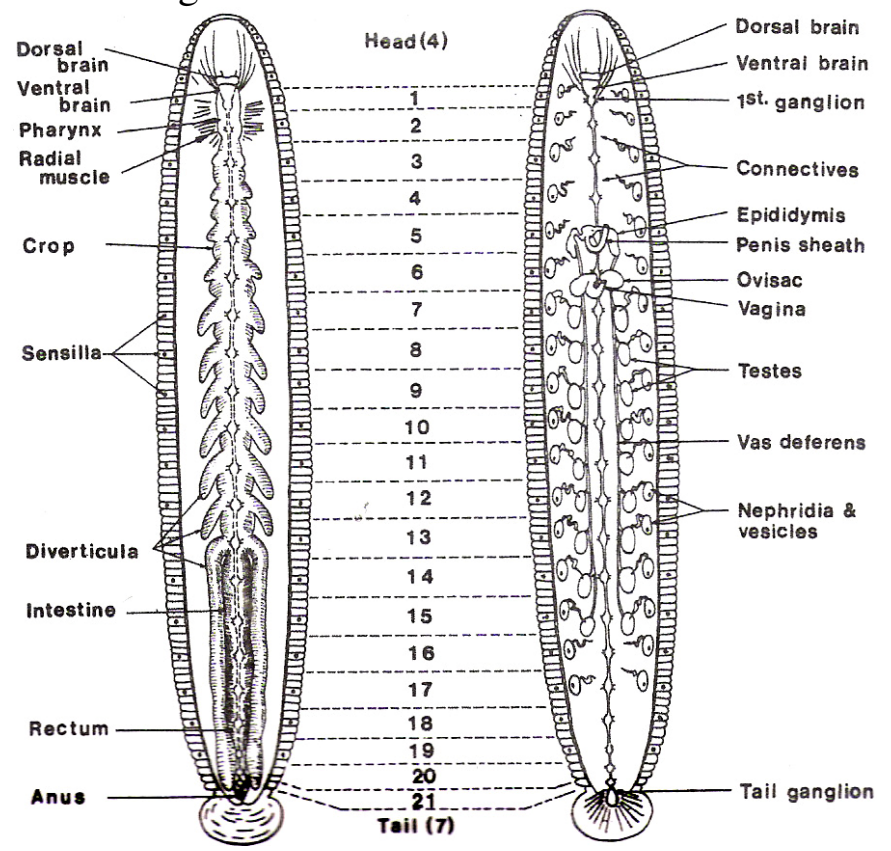

Figure 6. Full length leech anatomy diagram [1]. 
Appendix C: Ganglion Map and Index
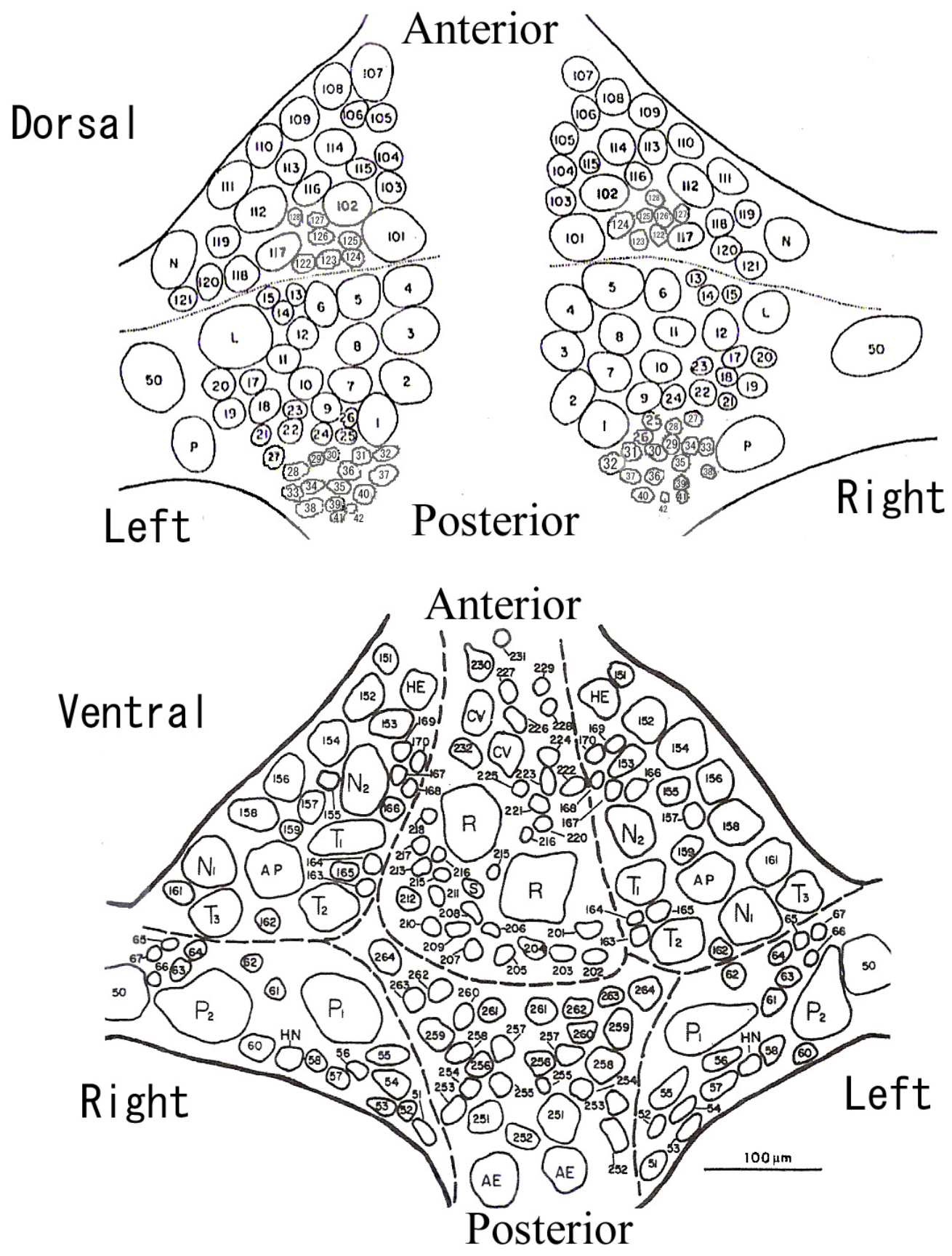

Figure 7. Ganglion cell location diagram [1] 


\begin{tabular}{|c|c|c|}
\hline$\underset{\#}{\text { Map }}$ & $\begin{array}{l}\text { Other } \\
\text { Designation }\end{array}$ & Description \\
\hline 1 & $I-I-d$ & inhibitor of dorsol longitudinal muscles \\
\hline 2 & $\mid-I-v$ & inhibitor of ventral longitudinal muscles \\
\hline 3 & & excitor of dorsomedial longitudinal muscles \\
\hline 4 & & excitor of ventromedial longitudinal muscles \\
\hline 5 & $\mathrm{dl}$ & excitor of dorsal longitudinal muscles \\
\hline 7 & & excitor of dorsal longitudinal muscles \\
\hline 8 & $\mathrm{vl}$ & excitor of ventral longitudinal muscles \\
\hline 11 & v3 & excitor of dorsal circular muscles \\
\hline 12 & $\mathrm{v} 1$ & excitor of ventrolateral circular muscles \\
\hline 17 & & excitor of dorsolateral longitudinal muscles \\
\hline 27 & & interneuron in swimming oscillator \\
\hline 28 & & interneuron in swimming oscillator \\
\hline 33 & & interneuron $\mathrm{n}$ in swimming oscillator \\
\hline 34 & $\mathrm{Cl}$ & $\begin{array}{l}\text { coupling interneuron; electrically couples T cells with } \mathrm{S} \text { cell and } \\
\text { contralateral cells; on ventral or dorsal surface interneron in } \\
\text { swimming oscillator }\end{array}$ \\
\hline 50 & Leydig & unknown function; putative neuosecretory cell \\
\hline 101 & Ifl & inhibitor of dorsoventral muscles \\
\hline 102 & & inhibitor of dorsal longitudinal muscles \\
\hline 106 & I & excitor of lateral longitudinal muscles \\
\hline 107 & $\mathrm{dl}$ & excitor of dorsomedial longitudinal muscles \\
\hline 108 & $\mathrm{v}$ & excitor of ventromedial longitudinal muscles \\
\hline 109 & $\mathrm{FI}$ & excitor of lateral dorsoventral muscles \\
\hline 110 & $\mathrm{Ob}$ & excitor of oblique muscles \\
\hline 111 & $\mathrm{Ob}$ & excitor of oblique muscles \\
\hline 112 & $d$ & excitor of dorsal circular muscles \\
\hline 117 & & excitor of medial dorsoventral muscles \\
\hline 119 & & inhibitor of ventral longitudinal muscles \\
\hline 123 & & interneuron in swimming oscillator \\
\hline 153 & & unknown function; receives excitatory photosensory input \\
\hline 154 & & unknown function; receives excitatory photosensory input \\
\hline 201 & & unknown function; inhibited by water vibration stimuli to sensilla \\
\hline 202 & & unknown function; inhibited by water vibration stimuli to sensilla \\
\hline 204 & & (unpaired) interneuron initiates and maintains swimming \\
\hline 205 & & $\begin{array}{l}\text { (unpaired) interneuron initiates and maintains swimming } \\
\text { (probably homologous with 104) }\end{array}$ \\
\hline
\end{tabular}

Table 2. Ganglion cell index [1]. 


\begin{tabular}{|c|c|c|}
\hline$\underset{\#}{\text { Map }}$ & $\begin{array}{l}\text { Other } \\
\text { Designation }\end{array}$ & Description \\
\hline 208 & & (unpaired) interneuron in swimming oscillator \\
\hline 215 & & unknown function; eceives excitatory photosensory input \\
\hline 216 & & unknown function; $\square$ eceives excitatory photosensory input \\
\hline 251 & nut & $\begin{array}{l}\text { unknown function; hyperpolarized by glutamate and } \mathrm{P} \text { cell } \\
\text { activity }\end{array}$ \\
\hline$A E$ & & excitor of subcutaneous muscles (annulus erector) \\
\hline CV & v2 & exitor of ventrolateral circular muscles \\
\hline $\mathrm{HE}$ & avocado & excitor of lateral heart tubes \\
\hline T1 & grape, $\mathrm{Tl}$ & mechanosensory, responds to touch on lateral third of skin \\
\hline T2 & grape, Tv & mechanosensory, responds to touch on ventral third of skin \\
\hline L & & $\begin{array}{l}\text { excitor of dorsal and vetral longitudinal muscles (large } \\
\text { longitudinal motor meuron) }\end{array}$ \\
\hline $\mathrm{HN}$ & & inhibitory interneuron in heart oscillator \\
\hline AP & A Pag & unknown function \\
\hline T3 & Plum, Td & mechanosensory, responds to touch on dorsal third of skin \\
\hline P1 & potato, $\mathrm{Pd}$ & mechanosensory, responds to pressing on dorsal skin \\
\hline P2 & potato, $\mathrm{Pv}$ & mechanosensory, responds to pressing on ventral skin \\
\hline N1 & cuke, Nv & $\begin{array}{l}\text { mechanosensory, responds to noxious stimuli on skin, } \\
\text { innervates peripheral HO cells }\end{array}$ \\
\hline N2 & SF, Nd & mechanosensory, responds to noxious stimuli on skin and gut \\
\hline $\mathrm{R}$ & Retzius, $\mathrm{Kz}$ & $\begin{array}{l}\text { effects mucus release, swimming, relaxation of longitudinal } \\
\text { muscles; contains } 5 \text {-hydroxytryptamine }(5-\mathrm{HT})\end{array}$ \\
\hline S & & $\begin{array}{l}\text { (Unpaired) interneuron contribes to shortening and swim } \\
\text { initiation; "giant" axon in Rohde's fiber }\end{array}$ \\
\hline
\end{tabular}




\section{Appendix D: Additional Ganglion Notes [1]}

- Each ganglion contains approximately 400 nerve cell Somas (ganglia 5 \& 6 contain approximately 700 cell bodies and control sex organs)

- Neurons are mono-polar

- Nerve somas are separated by septa into 6 groups within each ganglion

- Nerve soma diameters are approximately 10 - $60 \mu \mathrm{m}$

- Resting potential mainly governed by potassium ions

- resting potential approximately $-70 \mathrm{mV}$ 
Appendix E: Simplified Nerve Diagram

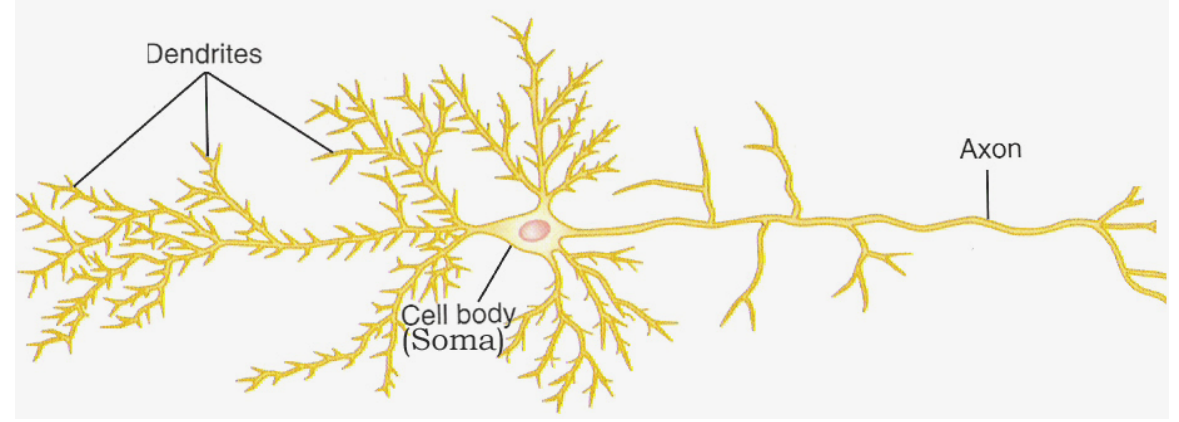

Figure 8. Simplified nerve diagram labeling key parts [2]. 
Appendix F: Laboratory List of Materials and Equipment

Dissection / Leech Care
10x - 50x Dissection Microscope
Petri Dishes
Candle Wax
Forceps (x2)
Dissection Scissors
Paper Towels
Leech's
Leech Solution
Leech Container
Pins

Data Collection

250x -500x Microscope

Gibraltar Table

Faraday Cage

Desktop Computer

Vibration Dampened Table

Multiclamp 700B (including headstages)

NI PCI6259 DAQ

Digidata $1440 \mathrm{~A}$

0.3M KCL

Flaming/Brown Micropipette Puller

Micropipette Tubes

Coaxal Cables

Bleach

Beakers

Table 3. Laboratory List of Materials and Equipment. 
Appendix G: Laboratory Equipment Connection Diagram

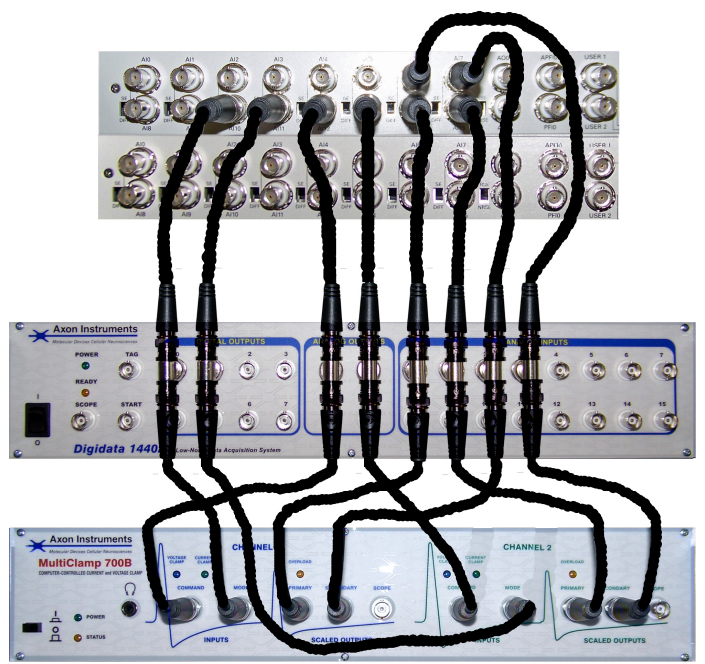

Figure 9. Wiring diagram of data acquisition hardware. 
Appendix H: Pipette Pulling Settings

\begin{tabular}{|l|l|l|l|}
\hline Glass Type: & O.D. $=1.0 \mathrm{~mm}$ & I.D. $=0.50 \mathrm{~mm}$ & Borosilicate Glass \\
\hline
\end{tabular}

\begin{tabular}{|c|c|c|c|c|}
\hline Heat & Pull & Velocity & Time & Pressure \\
\hline Ramp +10 & 100 & 100 & 200 & 400 \\
\hline
\end{tabular}

Table 4. Settings for pulling pipettes with the Flaming/Brown Micropipette Puller. 
Appendix I: Step-by-step procures for dissection of a ganglia (first method: removal of tissue)

\section{Leech Preparation}

1) $1-3$ leeches in a beaker of water

- use water from holding container

- fill beaker $1 / 3$ full and cover

2) Add alcohol, $2-3$ tea spoons

- 2 spoons: $35 \mathrm{~min}$ prep. time

- 3 spoons: $20 \mathrm{~min}$ prep. time

3) Wait until leech stops moving

Pinning The Leech

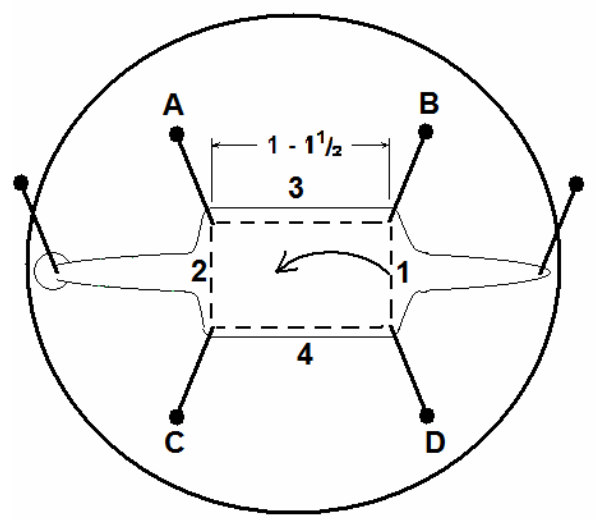

Figure 5. Diagram of leech pinning process.

1) Use Petri-dish $1 / 3$ full of Sylgard

2) Lay leech dorsal side up (dark-green side up, stripes up, mouth down)

3) Place two pins, one in each end of leech

- stretch leech to full diameter of dish

- if leech reacts to by constricting, the leech needs more time in alcohol

4) Place four pins, two pins on each side of $1-1 \frac{1}{2}$ inches apart

- stretch body until flat

\section{Cutting}

1) $\mathrm{Cut} \overline{\mathrm{AC}} \& \overline{\mathrm{BD}}$

2) $\mathrm{Cut} \overline{\mathrm{AB}} \& \overline{\mathrm{CD}}$

3) Use forceps to pull BD toward AC cutting all internal connections

4) Sever the back segment and discard

Locating the Ganglia

1) Dab a paper towel to remove excess blood from inside the leech

2) Locate the thin-long black line running laterally down the leech

3) Locate round bulges along the black line

- these house the ganglia

$-3-4$ are located in a $1-1 \frac{1}{2}$ inch section

4) Remove the sheath from around the ganglia

- sheath is black and fibrous

- ganglia is opaque

- puncture only the sheath, with forceps, near the edge

- hold ganglia with other forceps

- pull sheath away from the ganglia

- repeat this step until ganglia is fully visible 
Appendix J: Test Cell Schematic

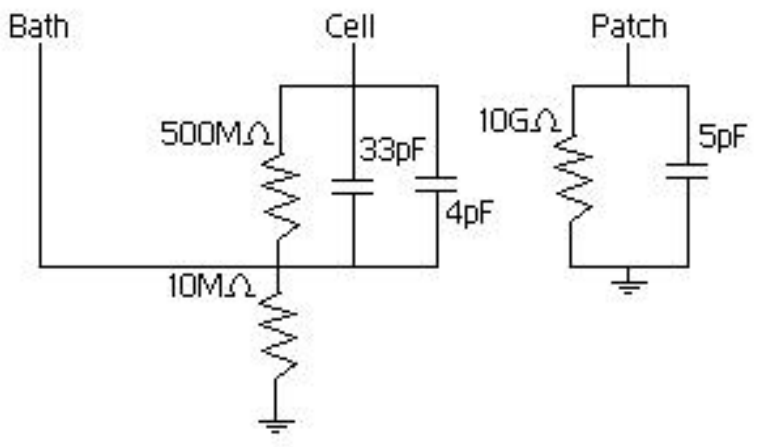

Figure 10. Axon instruments test cell schematic [8]. 


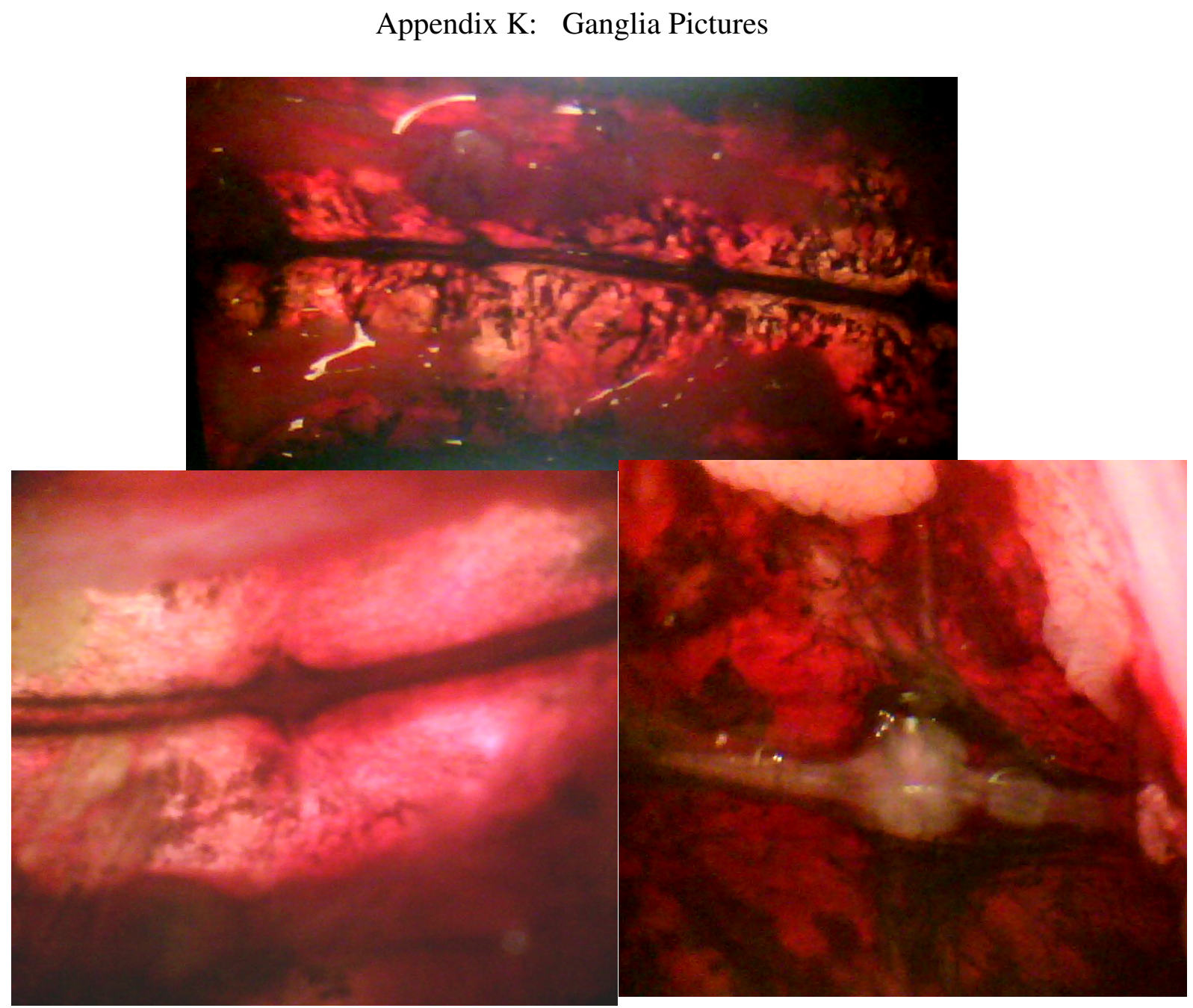

Figure 11. (Top) Group of ganglia, (Left) Single ganglia, (Right) Single ganglia with sheath removed. 
Appendix L: Dark Field Microscopy of Single Ganglion.

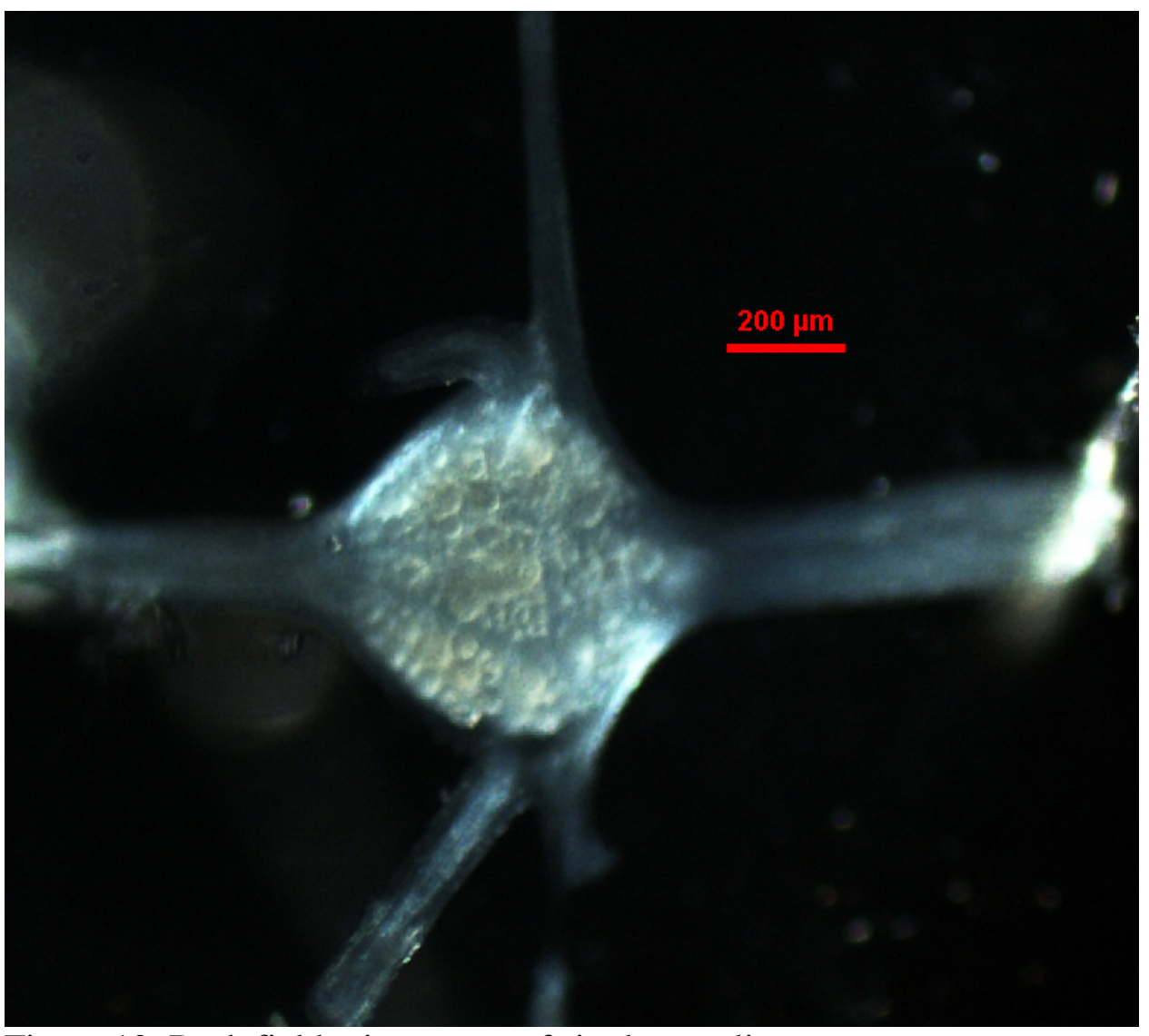

Figure 12. Dark field microscopy of single ganglion. 